unknown, though increased susceptibility to immunological injury in the elderly may be important. Interestingly in this respect, despite immediate withdrawal of piroxicam after the onset of jaundice in our patient evidence of severe liver damage persisted and was subsequently associated with hyperglobulinaemia and the formation of antinuclear antibodies in high titre (1/160). Possibly a self perpetuating autoimmune reaction triggered by piroxicam was responsible for the continued progression of the lesion to subacute hepatic necrosis.

A recent report from France described four patients with chronic active hepatitis due to clometacin (an indole derived non-steroidal anti-inflammatory drug widely used in France) which was associated with the formation of antidouble-stranded deoxyribonucleic acid antibodies. Withdrawal of the drug was followed by biochemical and clinical improvement with reduction in or disappearance of autoantibodies.

We find that corticosteroids are not useful in patients presenting with subacute hepatic failure. If this patient had been younger we would have considered liver transplantation at the time of transfer, when it was apparent that spontaneous recovery was very unlikely to occur.

1 Ludwig J, Axelsen R. Drug effects on the liver. An updated tabular compilation of drugs and drugrelated hepatic diseases. Dig Dis $S_{c i}$ 1983;28:651-60.

2 Brogden RN, Heel RC, Speight TM, Avery GS. Piroxicam: a review of its pharmacological properties and therapeutic efficacy. Drugs 1981;22:165-87.

3 Hartman H, Fischer G, Janning G. Prolonged cholestatic jaundice and leucopenia associated with piroxicam. $Z$ Gastroenterol 1984;22:343-5.

4 Taggart HM, Alderdice JM. Fatal cholestatic jaundice in elderly patients taking benoxaprofen. Br.Med f 1982;284:1372.

5 Caruana A, Andre C, Zafrani ES, Girardin MF, Metreau JM, Dhumcaux D. Serum antibodies against double-stranded DNA in chronic active hepatitis: an index of drug-induced liver disease? Lancet 1983;i:776.

Accepted 30 June 1986

Liver Unit, King's College Hospital, London SE5 9RS

$S$ M LEE, MB, MRCP, registrar

C J O'BRIEN, MB, MRCP, clinical research fellow

ROGER WILLIAMS, MD, FRCP, consultant physician and director

Epsom District Hospital, Surrey KT18 7EG

$S$ WHITAKER, MB, MRCP, registrar

S R GOULD, MD, MRCP, consultant physician

Correspondence to: Dr Williams.

\section{Programme for early detection of gastric cancer}

The low incidence of gastric cancer in the United Kingdom precludes such screening as is practised in Japan; however, high risk groups are identifiable. Morbidity and mortality statistics for consultations by general practitioners show that one in 50 patients presenting for the first time with dyspepsia will have gastric cancer. ${ }^{1}$ In this study a programme for the early detection of gastric cancer has been developed, based on examination of this selected population by endoscopy.

\section{Patients, methods, and results}

General practitioners from six practices were invited to refer all patients over the age of 40 presenting with dyspepsia to a dyspepsia clinic held in their health centre by members of the hospital team. After being interviewed and examined patients were informed that endoscopy was required to investigate their svmptoms. Endoscopy was performed at the hospital during the next week and thus a diagnosis was established within three weeks of the initial consultation. The referring doctor was informed of the diagnosis so that treatment could be begun. Patients with benign oesophageal and duodenal disease were not seen again. Those with gastric ulcers were re-examined to ensure healing at two months, and those with potentially precancerous histological changes were re-examined yearly. Patients with malignancy were referred for appropriate treatment.

Over two years 683 patients were referred. Forty five $(7 \%)$ failed to attend either the dyspepsia clinic or the appointment for endoscopy. We grouped the diagnoses as benign oesophageal disease including hiatus hernia and oesophagitis (198 patients), benign gastric disease including gastric ulceration and superficial gastritis (149 patients), and benign duodenal disease including duodenal ulceration and duodenitis (123 patients). In addition, there were 33 cases of malignancy, and 91 patients yielded normal results at endoscopy.

Fifteen $(2 \%)$ patients had gastric cancer (two had stage I cancer, five stage II, three stage III, and five stage IV), $10(67 \%)$ of whom were suitable for radical resection. All stage I lesions and two stage II lesions were diagnosed at follow up endoscopy. Three patients originally had gastric ulceration with adjacent intestinal metaplasia and one had atrophic gastritis. The remaining 18 cases of malignancy were either oesophageal tumours detected at endoscopy or cancers diagnosed by further investigation on the basis of clinical suspicion after endoscopy yielded normal results. There were seven oesophageal cancers, three colorectal cancers, four bronchial cancers, three cases of carcinomatosis, and one case of metastatic carcinoid.

A group with potentially precancerous histological changes was also identified. Seventy nine $(12 \%)$ had atrophic gastritis, intestinal metaplasia, or dysplasia alone or in combination. Twenty two had these changes adjacent to gastric ulcers but, as stated, four had a malignancy diagnosed during the study.

\section{Comment}

The rate of detection of gastric cancer in our study is similar to that predicted from mortality statistics for general practitioner consultation. The combination of specialised clinics in familiar surroundings and careful explanation of the examination contributed to the high compliance rate. The high rate of radical resection contrasts favourably with that of $18 \%$ from a previous regional survey. ${ }^{2}$ Equally encouraging was the proportion of lesions limited to the submucosa, which at $15 \%$ exceeds that of about $1 \%$ reported previously $^{3}$ and is comparable with the $30 \%$ detection rate reported from Japan. ${ }^{4}$

It remains to be seen whether follow up of those with premalignant changes will increase the number of patients with early gastric cancer. Further study is necessary to show whether the methods used in this pilot study are suitable for widespread use. The programme has been extended to include the Sandwell district of the West Midlands where the incidence of gastric cancer is higher (West Midlands Regional Cancer Registry, personal communication). This will enable prospective documentation of the true incidence of gastric cancer and evaluation of the financial implications of such a service.

WHA and MTH received grants from the Cancer Research Campaign during this study. We wish to thank the following general practitioners for entering their practices and patients into the study: Drs L A Pike, M E Clarkson, I G Cox, D L Crombie, C M Green, A Benn, M G Edward, W van Marle, K S Bhogal, M Simpson, D M Fleming, S R Sales, V S Tudor, D J Barford, A M Ross, A J Lucas, D L Kirk, S C S Meux, N Chauhan, J K Stewart, A J Campbell, P R Preston, D H Johnston, F E Campbell, M J Mortimer, J Kay, J Waters, C R Phillips, M H Lucy, and P P Borg-Bartolo.

1 Office of Population Censuses and Surveys. Mortality statistics for general practice. Second national study 1970-71. London: HMSO, 1972.

2 Fielding JWL, Roginski C, Ellis DJ, et al. Clinicopathological staging of gastric cancer. Br f Surg 1984;71:677-81.

3 Fielding JWL, Ellis DJ, Jones BG, et al. Natural history of "early" gastric cancer: results of a 10 year regional survey. BrMed f 1980;281:965-7.

4 Miwa K. Cancer of the stomach in Japan. Gann 1979;22:61-75.

(Accepted 25 fune 1986

Department of Surgery, Queen Elizabeth Hospital, Edgbaston, Birmingham

W H ALLUM, MD, FRCS, lecturer in surgery

$M$ T HALLISSEY, FRCS, research registrar

A DORRELL, SRN, research nurse

J LOW, SEN, research nurse

J W L FIELDING, MD, FRCS, consultant surgeon

Correspondence to: Mr Allum.

\section{Prognosis of patients discharged from a coronary care unit}

Previous work has shown that patients with and without acute myocardial infarction have a similar risk of death from coronary heart disease in the year after discharge from a coronary care unit. ${ }^{1.3}$ I present a retrospective study of survival of such patients over 10 years.

\section{Patients, methods, and results}

A total of 298 patients from 371 consecutive admissions to a coronary care unit who survived to 28 days were divided on discharge into two diagnostic categories (with and without acute myocardial infarction) according to the World Health Organisation electrocardiographic classification and serial measurements of serum creatine phosphokinase activity. Acute myocardial infarction was regarded as certain or probable when acute or non-acute ischaemic electrocardio- 